\title{
OS FUNDAMENTOS DA FíSICA DINÂMICA, DE WHITEHEAD (TRADUÇÃO AO PORTUGUÊS)
}

\author{
THE FOUNDATIONS OF DYNAMICAL PHYSICS, BY WHITEHEAD (TRANSLATION TO \\ PORTUGUESE)
}

Rafael Ferreira Martins ${ }^{1}$

\author{
Submetido: $24 / 02 / 2021$ \\ Aprovado: 17/03/2021
}

\section{RESUMO}

Este trabalho consiste na apresentação da pioneira primeira tradução ao português do capítulo 2 "Os Fundamentos da Física Dinâmica" (The Foundations of Dynamical Physics), incluindo seus dois apêndices ('As Leis de Newton do Movimento' e 'As Equações de Maxwell'), oriundo da parte 1 "As Tradições da Ciência" (The Traditions of Science), que integra a obra "Um Inquérito Concernente aos Princípios do Conhecimento Natural" (An Enquiry Concerning the Principles of Natural Knowledge) livro escrito pelo matemático e filósofo Alfred North Whitehead (1861 a 1947) na segunda década do século XX e publicado pela Editora da Universidade de Cambridge em 1919 (atualmente em domínio público). A justificativa para esta tradução é dada pela relevância da obra original, o livro que inaugurou a fase filosófica ampla da carreira de Whitehead, até então dedicada à fundamentação da lógica matemática. No capítulo aqui traduzido, especificamente, Whitehead expõe: reflexões sobre o embasamento teórico da mecânica clássica, relações da mecânica clássica com a teoria do éter material, implicações da pressuposição metafísica do éter material nas equações de Maxwell (e viceversa), explanações sobre as equações de Maxwell, críticas ao conceito de éter material e, finalizando, propõe o conceito de éter de eventos, em substituição ao éter tradicional. A fonte do texto original, utilizada para o trabalho de tradução, foi a versão digitalizada e disponibilizada online, para livre uso, pela Biblioteca da Universidade da Califórnia².

Palavras-chave: Whitehead. Tradução. Princípios do Conhecimento Natural. Tradições da Ciência. Fundamentos da Física Dinâmica.

\begin{abstract}
This paper presents a pioneering first translation into Portuguese of chapter 2 "The Foundations of Dynamic Physics", including its two appendices ('Newton's Laws of Motion' and 'Maxwell's Equations'), from part 1 "The Traditions of Science", which integrates the book "An Inquiry Concerning the Principles of Natural Knowledge" - book written by the mathematician and philosopher Alfred North Whitehead (1861 to 1947) in the second decade of the 20th century and published by the Cambridge University Publisher in 1919 (currently in public domain). The justification for this translation is given by the relevance of the original work, the book that inaugurated the broad philosophical phase of Whitehead's career, until then dedicated to the foundation of mathematical logic. In the specifically translated chapter, Whitehead exposes: reflections on the theoretical basis of classical mechanics, relations between classical mechanics and the theory of material ether, implications of the metaphysical assumption of material ether in Maxwell's equations (and vice versa), explanations about Maxwell's equations, critical to the concept of material ether and, finally, proposes the concept of 'event ether' to replace the traditional ether. The source for the original text, used during this translation work, was the version available online, for free use, by the University of California

Library.
\end{abstract}

\footnotetext{
1 Formado em Física (licenciatura) pela Universidade Paulista e formando em Filosofia (bacharelado) pela Universidade de Brasília. Membro do grupo de pesquisa 'Pensamento Processual e Estudos Whiteheadianos na América Latina' (UFRJ/CNPq) e integrante do projeto de pesquisa 'Whitehead e a Point-Free Geometry' (UnB). Agraciado com Menção Honrosa e indicado ao Prêmio Destaque de Iniciação Científica no XXV CIC UnB/DF. 2 WHITEHEAD, Alfred North. An Enquiry Concerning the Principles of Natural Knowledge. 1a Edição. Londres: Cambridge University $\quad$ Press, $1919 . \quad$ Disponível em: <https://archive.org/details/enquiryconcernpr00whitrich/page/n1/mode/2up>.
} 


\section{RECIMA21 - REVISTA CIENTÍFICA MULTIDISCIPLINAR}

OS FUNDAMENTOS DA FÍSICA DINÂMICA, DE WHITEHEAD (TRADUÇÃO AO PORTUGUÊS) Rafael Ferreira Martins

Keywords: Whitehead. Translation. Principles of Natural Knowledge. Traditions of Science. Foundations of Dynamical Physics.

\section{TRADUÇÃO}

\section{Um Inquérito Concernente aos Princípios do Conhecimento Natural \\ Parte 1: As Tradições da Ciência}

\section{Capítulo II: Os Fundamentos da Física Dinâmica}

\section{As Leis do Movimento de Newton}

4.1 As dificuldades teóricas encaminhadas pela aplicação da doutrina filosófica da relatividade nunca preocuparam os cientistas práticos. Eles começaram com os pressupostos funcionais ${ }^{1}$ de que, em algum sentido, o mundo está em um espaço euclidiano, que os pontos permanentes de tal espaço não possuem características individuais reconhecíveis por nós, exceto quando são ocupados por material reconhecível, ou exceto na medida em que são definidos por relações espaciais ordenadas ${ }^{2}$ por pontos que são, por sua vez, definitivamente reconhecíveis e, assim, de acordo com o propósito em questão, é possível assumir que a Terra ou qualquer outro eixo astronômico que for definido pelo auxílio do sistema solar, de estrelas e das considerações dinâmicas deduzidas das leis de Newton do movimento, está em repouso.

4.2 As leis do movimento de Newton pressupõem as noções de massa e força. A 'massa' surge da concepção de uma qualidade passiva de um corpo material, aquilo que está em si à parte de sua relação para com outros corpos; a noção de 'força' é a de uma agência ativa mudando as circunstâncias físicas de um corpo e, em particular, suas relações espaciais para com outros corpos. É francamente óbvio que massa e força foram introduzidas na ciência como resultado dessa antítese entre qualidade intrínseca e agência, embora reflexões adicionais possam, de alguma maneira, frustrar a simplicidade desta perspectiva. Massa e força são quantidades mensuráveis e suas expressões numéricas são dependentes das unidades escolhidas. A massa de um corpo é constante, contanto que o corpo permaneça composto pelo mesmo autoidêntico material. Velocidade, aceleração e força são quantidades vetoriais, isto é, possuem tanto direção quanto magnitude. Eles são, portanto, representáveis por linhas retas traçadas desde alguma origem arbitrária qualquer.

4.3 Essas leis do movimento estão entre as fundamentações da ciência e, certamente, qualquer alteração nelas deve ocorrer de modo a produzir efeitos observáveis apenas em circunstâncias muito excepcionais. Porém, como é muito frequente na ciência, um escrutínio de seus significados produz muitas perplexidades.

\footnotetext{
1 "pressupostos funcionais" foi a escolha de tradução, com base na melhor adequação contextual, para o original "working assumptions", cuja tradução direta (suposições de trabalho) poderia incorrer no risco de ambiguidade temática.

2 "ordenados" foi o termo escolhido para traduzir "assigned", uma vez que a tradução mais usual (atribuídos) asseguraria menos o conceito embutido no termo original.
} 


\section{RECIMA21 - REVISTA CIENTÍFICA MULTIDISCIPLINAR}

OS FUNDAMENTOS DA FÍSICA DINÂMICA, DE WHITEHEAD (TRADUÇÃO AO PORTUGUÊS)

Rafael Ferreira Martins

Em primeiro lugar, nós podemos colocar de lado uma dificuldade menor. Em nossa experiência, uma massa finita de matéria ocupa um volume e não um ponto. Evidentemente, para que as leis possam ser enunciadas de forma integral, envolverão, em certos pontos de exposição, uma maior elaboração do enunciado. Esses enunciados são expostos (com uma explicação um tanto abreviada) em tratados de dinâmica.

Em segundo lugar, a distinção de Lorentz, entre equações macroscópicas e equações microscópicas, impõe-se sobre nós, em razão da natureza molecular da matéria e da natureza dinâmica do calor. Um corpo aparentemente formado por matéria contínua, com suas relações geométricas intrínsecas quase invariáveis, é, de fato, composto por moléculas agitadas. As equações do movimento para tal corpo, conforme utilizadas por um engenheiro ou um astrônomo, são, na nomenclatura de Lorentz, macroscópicas. Nestas equações, mesmo um elemento diferencial de volume deve ser suposto como sendo suficientemente grande para representar a média ${ }^{3}$ das diversas agitações das moléculas e para registrar somente o resíduo geral desequilibrado que, para a observação ordinária, é o movimento do corpo.

As equações microscópicas são aquelas que se aplicam às moléculas individuais. É evidente, imediatamente, que uma série de tais conjuntos de equações é possível, na qual os conjuntos adjacentes são macroscópicos e microscópicos relativamente uns aos outros. Por exemplo, podemos penetrar molécula abaixo, até os elétrons e o núcleo que a compõe, e assim obter equações inframoleculares. É puramente uma questão de saber se há algum fenômeno observado que desse modo receba sua interpretação.

A evidência indutiva para a validação das equações de movimento de Newton, dentro dos limites experimentais de precisão, é obviamente muito mais forte no caso das equações macroscópicas do engenheiro e do astrônomo do que no caso das equações microscópicas da molécula, e ainda mais forte do que no caso das equações inframicroscópicas do elétron. Mas existe uma boa evidência de que mesmo as equações inframicroscópicas conformam-se com as leis de Newton em uma primeira aproximação. Os traços de desvio surgem quando as velocidades não são inteiramente insignificantes comparadas à da luz.

4.4 O que nós sabemos sobre massas e forças? Nós obtemos nosso conhecimento de forças tendo alguma teoria sobre massas, e nosso conhecimento de massas tendo alguma teoria sobre forças. Nossas teorias sobre as massas nos permitem, em certas circunstâncias, atribuir as razões numéricas das massas dos corpos envolvidos; então, os movimentos observados desses corpos nos permitirão registrar (pelo uso das leis do movimento de Newton) as direções e magnitudes das forças envolvidas e, a partir daí, formular teorias mais amplas quanto às leis que determinam a produção de força. Nossas teorias sobre a direção e as magnitudes comparativas das forças, com os movimentos observados dos corpos, permitir-nos-ão registrar (pelo uso das leis do movimento de Newton) as

\footnotetext{
3 "representar a média" foi a tradução escolhida para a ação "to average", pois a tradução mais literal do verbo "average" seria "mediar" e a tradução mais tematizada seria "ratear", porém a primeira opção alteraria a ideia original e a segunda acrescentaria uma ação inexistente.
} 


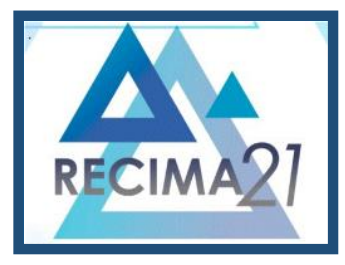

\section{RECIMA21 - REVISTA CIENTÍFICA MULTIDISCIPLINAR}

OS FUNDAMENTOS DA FÍSICA DINÂMICA, DE WHITEHEAD (TRADUÇÃO AO PORTUGUÊS) Rafael Ferreira Martins magnitudes comparativas das massas. Os resultados finais podem ser encontrados em livros de bolso de engenheiros, em tabelas de constantes físicas para físicos e em tabelas astronômicas. A verificação é a concordância dos resultados de experimentos diversos. Uma parte essencial de tais teorias é o julgamento de circunstâncias que são suficientemente análogas para garantir a suposição da mesma massa, ou da mesma magnitude de força, em diversos casos atribuídos. Ou seja, as teorias apoiam-se sobre o fato do reconhecimento.

4.5 Tem sido popular definir força como o produto da massa pela aceleração. A dificuldade a ser enfrentada com essa definição é que a familiar equação da dinâmica elementar, a saber,

$$
m \times a=F
$$

agora, torna-se

$$
m \times a=m \times a
$$

Não é fácil entender como uma ciência importante pode provir de tais premissas. Além disso, o simples equilíbrio de um peso pela tensão da mola de suporte recebe um significado muito artificial. Com a mesma razão, podemos começar com nossas teorias da força como fundamentais e definir a massa como a força dividida pela aceleração. Novamente, deveremos encontrar o mesmo perigo de reduzir equações dinâmicas a identidades, tais como

$$
\frac{F}{a}=\frac{F}{a}
$$

Igualmente, a massa permanente de uma barra de ferro recebe um significado muito artificial.

\section{O Éter}

5.1 A teoria do estresse ${ }^{4}$ entre corpos distantes, considerada um fato último, foi repudiada pelo próprio Newton, mas foi adotada por alguns de seus sucessores imediatos. No século XIX, a crença na ação à distância perdeu terreno gradativamente.

Existem quatro razões científicas definitivas para a adoção da teoria oposta, a da transmissão do estresse através de um meio intermediário, que chamaremos de 'éter'. Essas razões se somam às preferências filosóficas um tanto vagas, baseadas na desconexão envolvida na separação espacial e temporal. Em primeiro lugar, a teoria ondulatória da luz também postula um éter e, portanto, traz um testemunho simultâneo de sua existência. Em segundo lugar, Clerk Maxwell produziu as fórmulas para os estresses em tal éter, os quais, se existissem, seriam consideráveis para atrações gravitacionais, eletrostáticas e magnéticas. Nenhuma teoria da natureza do éter é produzida, o que de alguma forma explica por que tais estresses aí existem; e, portanto, sua existência é tão somente uma suposição desconectada, tanto quanto a dos estresses diretos entre corpos distantes. Em terceiro lugar, as equações do campo eletromagnético de Clerk Maxwell pressupõem eventos e propriedades físicas do espaço aparentemente vazio. Consequentemente, deve haver algo, ou seja, um éter, no espaço vazio, ao qual essas propriedades pertencem. Essas

\footnotetext{
4 "estresse" foi o termo escolhido, visando a manutenção do vocabulário filosófico do autor, para traduzir "stress", cujo conceito também pode ser entendido, no contexto das ciências físicas, perfeitamente como "tensão".
} 


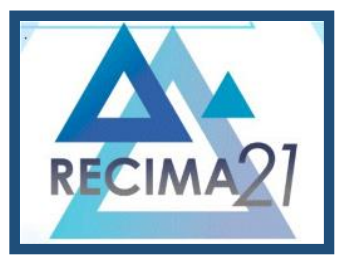

\section{RECIMA21 - REVISTA CIENTÍFICA MULTIDISCIPLINAR}

OS FUNDAMENTOS DA FÍSICA DINÂMICA, DE WHITEHEAD (TRADUÇÃO AO PORTUGUÊS)

Rafael Ferreira Martins equações são agora reconhecidas como os fundamentos da ciência exata do eletromagnetismo e estão no mesmo nível das equações do movimento de Newton. Assim, outro testemunho é adicionado à existência de um éter.

Por último, a identificação da luz com as ondas eletromagnéticas, feita por Clerk Maxwell, mostra que o mesmo éter é exigido pelos aparentemente diversos fenômenos ópticos e eletromagnéticos. É removida a objeção de que novas propriedades devem ser atribuídas ao éter por cada uma das distintas linhas de pensamento que o postulam.

Será observado que a gravitação está fora desta unificação da teoria científica devida ao trabalho de Maxwell, ao menos até conhecermos os estresses no éter que a produziriam.

5.2 A suposição da existência de um éter levanta imediatamente a questão acerca de suas leis do movimento. Portanto, em adição à hierarquia das equações macroscópicas e microscópicas, existem, de outra forma, as equações de movimento para o éter no espaço vazio. As razões a priori para acreditar que as leis do movimento de Newton se aplicam ao éter são muito fracas, sendo, na verdade, nada mais do que a extensão indutiva dessas leis para casos amplamente dissimilares daqueles nos quais foram verificadas. Não obstante, é um procedimento científico sadio investigar se as propriedades assumidas do éter são explicáveis na suposição de que ele está se comportando como matéria comum, mesmo que apenas para obter sugestões por contraste, para a formulação das leis que expressam suas mudanças físicas.

O melhor método para proceder é assumir certos grandes princípios dedutíveis das leis de Newton e interpretar certos vetores eletromagnéticos como deslocamentos e velocidades do éter. Deste modo, Larmor teve sucesso em deduzir as equações de Maxwell a partir do princípio da menor ação após fazer as suposições necessárias. Nisso, ele está apenas seguindo uma longa série de cientistas anteriores que, durante o século XIX, dedicaram-se à explicação dos fenômenos ópticos e eletromagnéticos. Seu trabalho completa um século de realizações notáveis neste campo.

5.3 É possível questionar, entretanto, se esse procedimento não é uma inversão da linha de pensamento mais fundamental. Deve-se notar que as equações de Newton, ou quaisquer princípios equivalentes que as substituam, são, em algum sentido, apenas formas em branco. Elas precisam ser complementadas por hipóteses a respeito da natureza dos estresses, das massas e dos movimentos antes que possa haver qualquer possibilidade de sua aplicação. Assim, no momento em que as equações de movimento de Newton são aplicadas à explicação de eventos etéreos, há um grande acúmulo de hipóteses a respeito de coisas das quais sabemos muito pouco. O que de fato sabemos sobre o éter está resumido nas equações de Maxwell ou em adaptações recentes de suas equações, como as devidas a Lorentz. A descoberta da massa eletromagnética e do momento eletromagnético sugere que, ao menos para o éter, ganhamos concepções mais simples dos fatos tomando as equações de Maxwell, ou as equações de Lorentz-Maxwell, como fundamentais. Essas equações seriam, então, as equações microscópicas finais, pelo menos no estágio atual da ciência, e as equações de Newton se tornariam equações macroscópicas, que se aplicam em certas 


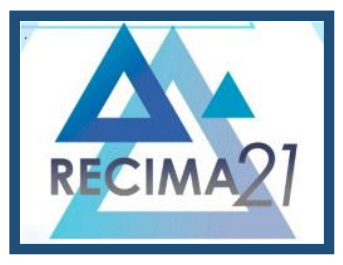

\section{RECIMA21 - REVISTA CIENTÍFICA MULTIDISCIPLINAR}

\section{OS FUNDAMENTOS DA FÍSICA DINÂMICA, DE WHITEHEAD (TRADUÇÃO AO PORTUGUÊS) \\ Rafael Ferreira Martins} circunstâncias definidas a agregados etéreos. Tal procedimento não pré-julga a teoria debatida da origem puramente eletromagnética da massa.

5.4 A teoria molecular moderna é destruidora da obviedade do pré-julgamento em favor dos conceitos tradicionais de material último em um instante. Considere uma molécula de ferro. Ela é composta por um núcleo central, de eletricidade positiva, rodeado por aglomerados anulares de elétrons, compostos de eletricidade negativa e girando em torno do núcleo. Nenhuma propriedade característica do ferro pode ser manifestada em um instante. Instantaneamente, há apenas uma distribuição de eletricidade e as equações de Maxwell para expressar nossas expectativas. Mas o ferro não é uma expectativa, nem mesmo uma memorização. É um fato; e esse fato, que é o ferro, é o que acontece durante um período de tempo. O ferro e um organismo biológico estão em um nível que requer tempo para funcionar. Não existe ferro em um instante; ser ferro é a característica de um evento. Toda constante física a respeito do ferro, que aparece nas tabelas científicas, é o registro de tal caráter. O que há de último no ferro, de acordo com a teoria tradicional, são as distribuições instantâneas de eletricidade; e essa ultimidade ${ }^{5}$ é atribuída simplesmente em razão de uma teoria metafísica, e não por motivo de observação.

5.5 $\mathrm{Na}$ verdade, quando admitimos a hierarquia das equações macroscópicas e microscópicas, o conceito tradicional se perdeu, pois são as equações macroscópicas que expressam os fatos da observação imediata, e essas equações expressam essencialmente as características integrais dos eventos. Essa hierarquia, todavia, é necessária para todos os conceitos da física moderna - a teoria molecular da matéria, a teoria dinâmica do calor, a teoria das ondas da luz, a teoria eletromagnética das moléculas, a teoria eletromagnética da massa.

\section{As Equações de Maxwell ${ }^{6}$}

6.1 Uma discussão acerca das equações de Maxwell constituiria um tratado sobre eletromagnetismo. Porém, elas exemplificam algumas considerações gerais sobre leis físicas.

Estas equações (expressas para um sistema de coordenas $\alpha$ ) envolvem, para cada ponto do

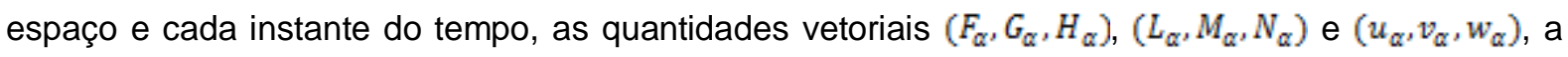
saber, as 'forças' elétrica e magnética e a velocidade da carga elétrica. Agora, um vetor envolve direção, e a direção não está preocupada meramente com o que está naquele ponto. É impossível definir direção sem referência ao resto do espaço; especificamente, envolve alguma relação com o todo do espaço.

Novamente, as equações envolvem os operadores diferenciais espaciais $\frac{\partial}{\partial_{x_{\pi}}}, \frac{\partial}{\partial_{y_{\pi}}}, \frac{\partial}{\partial_{x_{\pi}}}$, que são representados pelos símbolos $\operatorname{rot}_{\alpha}$ e diviva ; e elas também envolvem o operador diferencial temporal $\frac{\partial}{\partial_{t_{\alpha}}}$. Desta maneira, os coeficientes diferenciais produzidos expressam essencialmente propriedades na vizinhança do ponto $\left(x_{\mathbb{K}^{*}}, y_{\kappa}, z_{\alpha}\right)$ e do tempo $t_{\alpha}$, e não apenas propriedades em

\footnotetext{
5 "ultimidade" foi o neologismo construído para representar, em língua portuguesa, o conceito whiteheadiano "ultimateness", empregado no texto original.

${ }^{6}$ Vide apêndice 2 deste capítulo, também incluído neste trabalho de tradução.
} 


\section{RECIMA21 - REVISTA CIENTÍFICA MULTIDISCIPLINAR}

OS FUNDAMENTOS DA FÍSICA DINÂMICA, DE WHITEHEAD (TRADUÇÃO AO PORTUGUÊS)

Rafael Ferreira Martins $\left(x_{\alpha^{v}} y_{\alpha}, z_{\alpha^{x}}, t_{\alpha}\right)$, pois um coeficiente diferencial é um limite, e o limite de uma função em um determinado valor de seu argumento expressa uma propriedade da agregação dos valores da função correspondente à agregação dos valores do argumento na vizinhança do valor dado.

Este é essencialmente o mesmo argumento expresso anteriormente em 1.2 para o caso particular do movimento. Quer dizer, não podemos expressar os fatos da natureza como um agregado de fatos individuais em pontos e instantes.

6.2 Nas equações de Lorentz-Maxwell ${ }^{7}$, não há referência ao movimento do éter. A velocidade $\left(u_{\alpha}, v_{\alpha}, w_{\alpha}\right)$ que aparece neles é a velocidade da carga elétrica. Quais são então as equações do movimento do éter? Antes de trabalharmos sobre essa questão, surge uma dúvida preliminar. $\mathrm{O}$ éter se move?

Se a ciência deve se basear nos dados incluídos nas equações de Lorentz-Maxwell, mesmo que as equações sejam modificadas, certamente, o movimento do éter não entra na experiência. Assim, Lorentz assume um éter estacionário: um éter sem movimento, que é simplesmente a entidade última da qual os vetores $\left(F_{\alpha}, G_{\alpha}, H_{\alpha}\right)$ e $\left(L_{\alpha}, M_{\alpha}, N_{\alpha}\right)$ expressam propriedades. Esse éter certamente tem uma existência muito obscura; e, no entanto, não podemos presumir que ele se move, apenas para lhe dar algo para fazer.

6.3 Os fatos últimos contemplados nas equações de Maxwell são as ocorrências de $P_{\mathscr{\alpha}}$

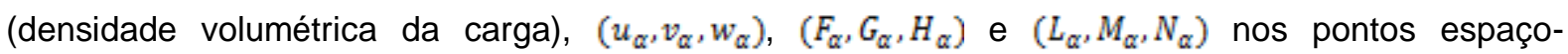
temporais da vizinhança ao redor do ponto espaço-temporal ( $x_{\alpha^{v}} y_{\alpha^{s}} z_{\alpha^{v}} t_{\alpha}$ ). Isso, porém, é apenas para dizer que os fatos últimos contemplados pelas equações de Maxwell são certos eventos que estão ocorrendo em todo o espaço. O material chamado de éter é apenas o resultado de um desejo metafísico. A continuidade da natureza é a continuidade dos eventos; e a doutrina da transmissão deve ser interpretada como uma doutrina da coextensividade dos eventos com espaço e tempo, bem como de sua interação recíproca. Nesse sentido, um éter pode ser admitido; mas, tendo em vista a implicação do termo existente, a clareza é obtida por uma distinção de fraseologia. Devemos denominar o éter tradicional como 'éter de matéria' ou 'éter material', e devemos empregar o termo 'éter de eventos' para expressar a suposição deste inquérito, que pode ser vagamente declarada como sendo 'que algo está acontecendo em todos os lugares e sempre'. É nosso propósito expressar com precisão as relações entre esses eventos na medida em que eles são revelados por nossa experiência perceptiva e, em particular, considerar aquelas relações das quais os conceitos essenciais de Tempo, Espaço e material persistente são derivados. Para tal, primeiramente, não devemos conceber os eventos como ocorrendo em um determinado Tempo, em um determinado Espaço e consistindo em mudanças de determinado material persistente. Tempo, espaço e material são adjuntos de eventos. Na velha teoria da relatividade, Tempo e Espaço são relações entre materiais; em nossa teoria, eles são relações entre eventos.

\footnotetext{
7 Vide apêndice 2 deste capítulo, também incluso neste trabalho de tradução.
} 


\section{RECIMA21 - REVISTA CIENTÍFICA MULTIDISCIPLINAR}

\section{Apêndice I ao Capítulo II}

\section{As Leis do Movimento de Newton}

Consideremos $\left(O_{\alpha}, X_{\alpha}, Y_{\alpha}, Z_{\alpha}\right)$, como na figura a seguir, eixos ortogonais em repouso;

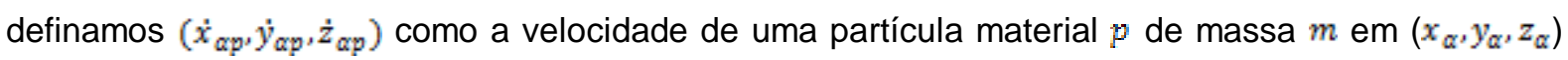
relativa a esses eixos, e tenhamos $\left(\underline{x}_{\kappa p}, \ddot{y}_{\kappa p}, z_{a p}\right)$ como a aceleração da mesma partícula. Ainda, consideremos $\left(X_{\alpha p}, Y_{a p}, Z_{a p}\right)$ como a força na partícula $p$. As duas primeiras leis de Newton podem ser compactadas nas equações:

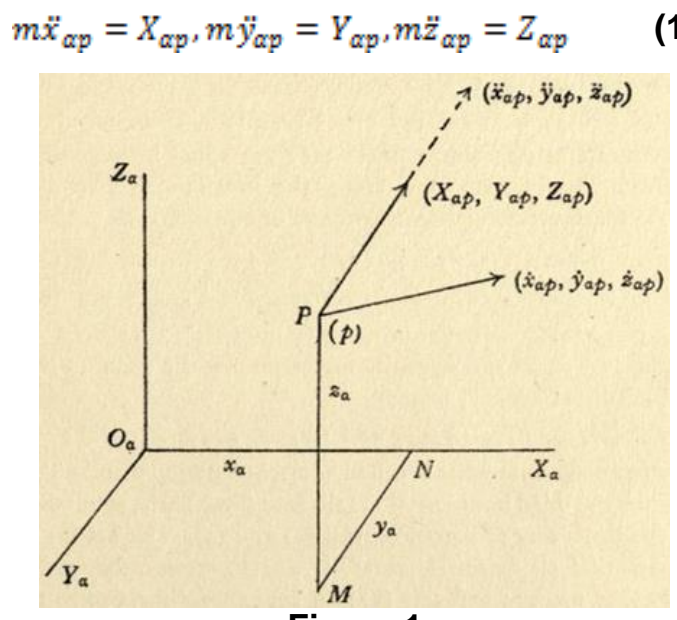

Figura 1

É desnecessário traçar as consequências elementares dessas equações.

A terceira lei do movimento considera uma característica fundamental da força e está fundamentada no sólido princípio de que toda agência nada mais é do que relações entre as entidades que estão entre os dados últimos da ciência. A lei é: Ação e reação são iguais e opostas. Isso significa que deve haver partículas $p^{s}{ }_{0} p^{t s}{ }^{t s} p^{t s p}$, etc. às quais a agência $\left(X_{\alpha p}, Y_{a p}, Z_{a p}\right)$ é devida, e isso nós podemos escrever como

$$
\left\{\begin{array}{l}
X_{a p}=X_{a p p^{\prime}}+X_{a p p^{n}}+\cdots \\
Y_{a p}=Y_{\alpha p p^{\prime}}+Y_{\alpha p p^{n}}+\cdots \\
Z_{a p}=Z_{a p p^{\prime}}+Z_{a p p^{n}}+\cdots
\end{array}\right\}
$$

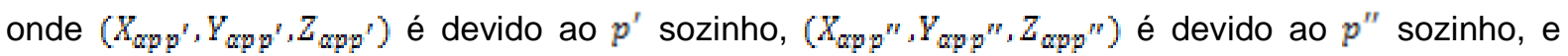
assim por diante.

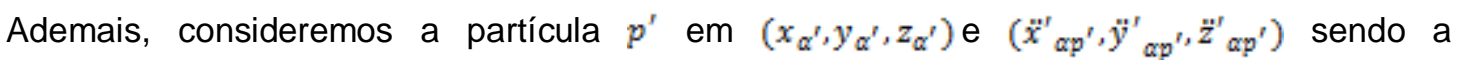
aceleração de $p^{s}$. Também, deixemos $\left(X_{\alpha p^{p},} Y_{\alpha p^{s},} Z_{\alpha p^{s}}\right)$ sendo a força em $p^{s}$; e $X_{\alpha p^{s} p^{s}} Y_{\alpha p^{\prime} p}$, etc. tendo significados para $p^{s}$ análogos àqueles que $X_{\text {app }}$, etc. tem para $p$. Então, de acordo com a terceira lei, 


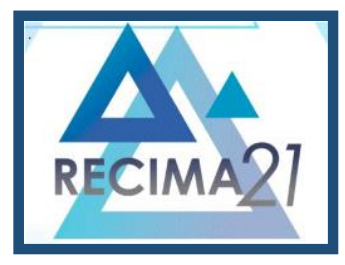

\section{RECIMA21 - REVISTA CIENTÍFICA MULTIDISCIPLINAR}

OS FUNDAMENTOS DA FÍSICA DINÂMICA, DE WHITEHEAD (TRADUÇÃO AO PORTUGUÊS)

Rafael Ferreira Martins

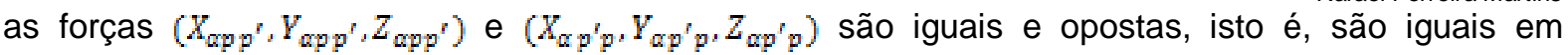
magnitude, opostas em sentido ${ }^{8}$ e estão sobre a mesma linha que junta $p$ e $p^{*}$. Esses requisitos resultam em dois conjuntos de equações

$$
\begin{aligned}
& X_{\alpha p p^{\prime}}+X_{\alpha p^{\prime} p}=0, Y_{\alpha p p^{\prime}}+Y_{\alpha p^{\prime} p}=0, Z_{\alpha p p^{\prime}}+Z_{\alpha p^{\prime} p}=0 \\
& \left(y_{\alpha} Z_{\alpha p p^{\prime}}-z_{\alpha} Y_{\alpha p p^{\prime}}\right)+\left(y_{\alpha^{\prime}} Z_{\alpha p^{\prime} p}-z_{\alpha^{\prime}} Y_{\alpha p^{\prime} p}\right)=0
\end{aligned}
$$

com duas equações análogas.

As duas forças iguais e opostas em $p$ e $p^{s}$, devido à sua agência direta mútua, a saber, $\left(X_{\alpha p p^{\prime},} Y_{\alpha p p^{\prime},} Z_{a p p^{\prime}}\right)$ e $\left(X_{\alpha p^{\prime} p}, Y_{\alpha p^{\prime} p}, Z_{\alpha p^{\prime} p}\right)$, conjuntamente constituem o que é chamado de "estresse" entre $p$ e $p^{s}$.

Assim, a terceira lei do movimento divide-se em três partes, simbolizadas pelos três conjuntos de equações (2), (3) e (4). O conjunto (2) expressa que toda força sobre a matéria é devida a estresses entre ela e outra matéria; e os conjuntos (3) e (4) expressam as duas características fundamentais dos estresses. Não precisamos parar para indagar se a curta expressão verbal da lei expressa logicamente essas três propriedades. Esse é um ponto menor de exposição, dependente do contexto em que esta formulação da lei se encontra.

\section{Apêndice II ao Capítulo II \\ As Equações de Maxwell}

Será conveniente apresentar essas equações em uma forma levemente modificada, a qual é devida à Lorentz. O espaço está referido pelo sistema fixo de eixos ortogonais $\alpha$, como no subartigo 6.1. Será necessário explicar alguns pormenores quanto a nomenclatura e notação.

Um vetor é uma quantidade física direcionada; por exemplo, a força elétrica em um ponto é um vetor. Este exemplo também mostra que devemos conceber vetores que têm significados análogos em diferentes pontos do espaço. Tal vetor é a força elétrica que pode ter magnitude e direção distintas em cada ponto do espaço, mas expressa em todos os pontos um fato físico definido. Tal vetor será uma função de sua posição, isto é, das coordenadas do ponto $\left(x_{\tilde{\alpha}}, y_{\alpha}, z_{\alpha}\right)$ do qual é característico aquele vetor.

Seja $\left(X_{\alpha}, Y_{\alpha}, Z_{\alpha}\right)$ um desses vetores. Então, $X_{\alpha}, Y_{\alpha}$ e $Z_{\alpha}$ são, cada um deles, funções de $\left(x_{\alpha^{\prime}}, y_{\alpha^{\prime}}, z_{\alpha}\right)$ e, ainda, do tempo $t_{\alpha}$, ou seja, eles são funções de $x_{\mathbb{\alpha}^{v}}, y_{\alpha^{\prime}} z_{\mathbb{\alpha}^{\prime}}, t_{\alpha}$. Nós devemos assumir que nossas quantidades físicas são diferenciáveis, exceto, possivelmente, em pontos excepcionais.

Conceda $q\left(X_{\mathscr{\alpha}}, Y_{\tilde{\alpha}}, Z_{\alpha}\right)$ para representar $\left(q X_{\tilde{\alpha}}, q Y_{\alpha}, q Z_{\alpha}\right)$ e, analogamente,

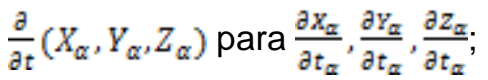

$$
\begin{aligned}
& \operatorname{div}_{\alpha}\left(X_{\alpha}, Y_{\alpha}, Z_{\alpha}\right) \text { para } \frac{\partial x_{\tilde{\alpha}}}{\partial x_{\alpha}}, \frac{\partial Y_{\alpha}}{\partial y_{\alpha}}, \frac{\partial z_{\alpha}}{\partial z_{\alpha}},
\end{aligned}
$$

8 No texto original foi empregada a palavra "direction" ("direção") como um conceito que, segundo as convenções matemáticas atuais, é especificamente o de "sentido", logo, optou-se pela tradução mais contextualizada, para evitar enganos conceituais. 


\section{RECIMA21 - REVISTA CIENTÍFICA MULTIDISCIPLINAR}

OS FUNDAMENTOS DA FÍSICA DINÂMICA, DE WHITEHEAD (TRADUÇÃO AO PORTUGUÊS)

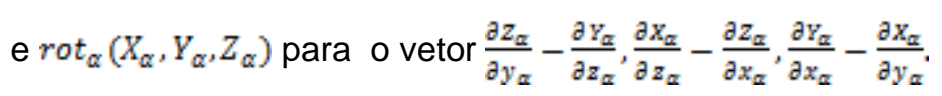

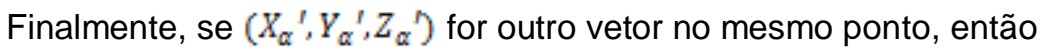

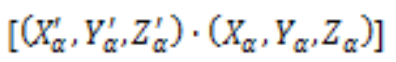

representa o que é chamado de 'produto vetorial' dos dois vetores, ou seja, o vetor

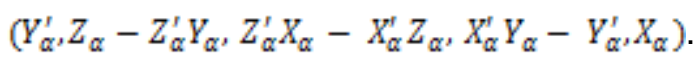

É evidente que o $\operatorname{rot}_{\alpha}\left(X_{\alpha}, Y_{\alpha}, Z_{\alpha}\right)$ pode ser expresso na forma simbólica

$$
\left[\left(\frac{\partial}{\partial x_{\alpha}}, \frac{\partial}{\partial y_{\alpha}}, \frac{\partial}{\partial z_{\alpha}}\right) \cdot\left(X_{\alpha,} Y_{\alpha}, Z_{\alpha}\right)\right] \text {. }
$$

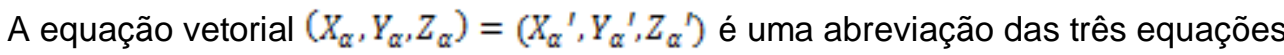

$$
X_{\alpha}=X_{\alpha, s}^{s} Y_{\alpha}=Y_{\alpha, s}^{s} Z_{\alpha}=Z_{\alpha}^{s} \text {. }
$$

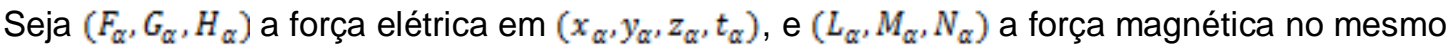
ponto e tempo. Também, sendo $\rho_{\alpha}$ a densidade volumétrica da carga magnética e $\left(u_{\alpha_{\alpha}}, v_{\alpha}, w_{\alpha}\right)$ sua respectiva velocidade; e considerando $\left(P_{\alpha}, Q_{\alpha}, R_{\alpha}\right)$ como a força ponderomotriz: todos igualmente em $\left(x_{\alpha} y_{\alpha}, z_{\alpha}, t_{\alpha}\right)$. Finalmente, sendo $c$ a velocidade da luz no vácuo.

Então, a forma de Lorentz para as equações de Maxwell é

$$
\begin{aligned}
& \operatorname{div}_{\alpha}\left(F_{\alpha}, G_{\alpha}, H_{\alpha}\right)=\rho_{\alpha} \\
& \operatorname{div}_{\alpha}\left(L_{\alpha}, M_{\alpha}, N_{\alpha}\right)=0 \\
& \operatorname{rot}_{\alpha}\left(L_{\alpha}, M_{\alpha}, N_{\alpha}\right)=\frac{1}{c}\left\{\frac{\partial}{\partial t}\left(F_{\alpha^{x}}, G_{\alpha}, H_{\alpha}\right)+\rho_{\alpha}\left(u_{\alpha}, v_{\alpha}, w_{\alpha}\right)\right\} \\
& \operatorname{rot}_{\alpha}\left(F_{\alpha}, G_{\alpha}, H_{\alpha}\right)=\frac{1}{c} \frac{\partial}{\partial t}\left(L_{\alpha}, M_{\alpha}, N_{\alpha}\right)
\end{aligned}
$$

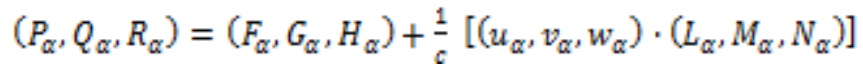

Deve-se notar que cada uma das equações vetoriais (3), (4) e (5) representa três equações ordinárias, de modo que há onze equações nas cinco fórmulas. 